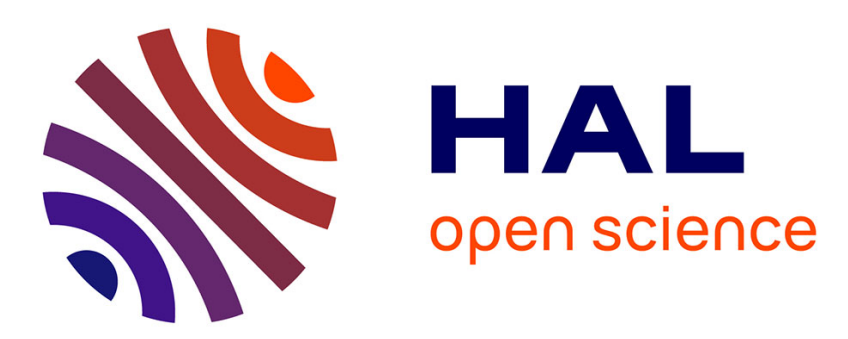

\title{
Classification of topologically stable defects in ordered media
}

\author{
M. Kléman, L. Michel, G. Toulouse
}

\section{To cite this version:}

M. Kléman, L. Michel, G. Toulouse. Classification of topologically stable defects in ordered media. Journal de Physique Lettres, 1977, 38 (10), pp.195-197. 10.1051/jphyslet:019770038010019500 . jpa00231358

\section{HAL Id: jpa-00231358 https://hal.science/jpa-00231358}

Submitted on 1 Jan 1977

HAL is a multi-disciplinary open access archive for the deposit and dissemination of scientific research documents, whether they are published or not. The documents may come from teaching and research institutions in France or abroad, or from public or private research centers.
L'archive ouverte pluridisciplinaire HAL, est destinée au dépôt et à la diffusion de documents scientifiques de niveau recherche, publiés ou non, émanant des établissements d'enseignement et de recherche français ou étrangers, des laboratoires publics ou privés. 


\title{
CLASSIFICATION OF TOPOLOGICALLY STABLE DEFECTS IN ORDERED MEDIA
}

\author{
M. KLÉMAN \\ Laboratoire de Physique des Solides \\ Université Paris Sud, 91405 Orsay, France \\ L. MICHEL $\left({ }^{*}\right)$ \\ Randall Laboratory of Physics \\ University of Michigan, Ann Arbor, MI 48109 U.S.A. \\ and \\ G. TOULOUSE \\ Groupe de Physique des Solides \\ Ecole Normale Supérieure, 75231 Paris Cedex 05, France
}

(Reçu le 14 mars 1977, accepté le 21 avril 1977)

\begin{abstract}
Résumé. - Les défauts de symétrie d'un milieu ordonné qui sont topologiquement stables, sont classés par des groupes d'homotopie. A titre d'exemple, nous établissons une classification complète de ces défauts de symétrie cristalline.
\end{abstract}

Abstract. - Topologically stable symmetry defects in ordered media can be classified by some
homotopy groups. As an example we establish a complete classification of such defects for crystals.

Two of us, G. T. and M. K., have proposed [1] a classification of elementary stable defects by the homotopy groups of the manifold of states. In this letter we will give a more systematic approach and present some applications of this classification, particularly for crystals.

1. - Let $\mathrm{G}$ be the symmetry group of the physical laws governing a thermodynamical system $\mathrm{M}$. Among the statistical equilibrium states of $M$, there generally exist not only a phase with symmetry G, but also other phases with less symmetry : in a perfect state (i.e., without defects, and idealized as an infinite system) such a phase is invariant under a subgroup $\mathrm{H}$ of $\mathrm{G}$; the action of $G$ on this state yields an orbit $G / H$ of states with identical physical properties; this orbit is the manifold of states of ref. [1]. If there are defects, $\mathrm{H}$ invariance is only local : different domains of $\mathrm{M}$ correspond to different points of the orbit $\mathrm{G} / \mathrm{H}$ (i.e., these different local states are transformed into each other by G). Ideally, this situation defines a function $\varphi$, taking its values in $\mathrm{G} / \mathrm{H}$, at each point of the conti-

$\left(^{*}\right)$ Permanent address : I.H.E.S., 91440 Bures sur Yvette, France. nuous medium outside the defects. (These considerations also apply to any model of statistical mechanics in a space of dimension $d$.) The restriction of the function $\varphi$ to an $r$-dimensional sphere $S_{r}$ defines a homotopy class $\alpha_{r} \varepsilon \pi_{r}(\mathrm{G} / \mathrm{H})$. If $\alpha_{r}=0, \varepsilon \pi_{r}$, the function $\varphi$ can be extended to an $(r+1)$-dimensional disc bounded by $S_{r}$ and, on this (contractible) disc, this extended function is homotopic to a constant, i.e., to the function of the perfect state. When $\alpha_{r} \neq 0$, $S_{r}$ encloses a topologically stable defect of dimension $d^{\prime}=d-1-r$. If $\alpha_{r}=0$ for all $r$ such that

$$
0 \leqslant r \leqslant d-1 \text {, }
$$

then by continuous deformation the state of the system can be made perfect in the interior of the sphere $S_{d-1}\left(d=3\right.$ in our space). When some $\alpha_{r}$ are nontrivial, there is a topologically stable defect (= t.s. defect in this letter).

We are naturally led to a classification of t.s. defects; of course such a classification cannot depend on the base point chosen for computing the homotopy classes. In all applications $\mathrm{G}$ will be a Lie group (which might be of dimension zero, i.e., discrete); when $\mathrm{G}$ and $\mathrm{H}$ are connected, $\mathrm{G} / \mathrm{H}$ is $n$-simple for 
every $n$ (see ref. [2], theorem 16.11); then the t.s. defects of dimension $d^{\prime}$ can be classified by the elements of $\pi_{r}(\mathrm{G} / \mathrm{H})$ with $r=d-1-d^{\prime}$. When $\mathrm{H}$ is not connected, $\pi_{1}(\mathrm{G} / \mathrm{H})$ may act nontrivially on $\pi_{n}(G / H)$ and the t.s. defects will be classified by the orbits of $\pi_{1}$ in $\pi_{n}$; for instance if $\pi_{1}$ is not abelian, the line defects (when $d=3$ ) will be classified by the conjugation classes of $\pi_{1}$.

In ref. [1], these ideas have been applied to several systems, e.g., superfluid ${ }^{4} \mathrm{He}$ and ${ }^{3} \mathrm{He}$. For all applications here, $\mathrm{G}=\mathrm{E}(3)$ the Euclidean group in 3dimensions. It is the semi-direct product $R_{\square}^{3} O(3)$ of the translation group $\mathrm{R}^{3}$ by $\mathrm{O}(3)$. We denote by $\mathrm{E}_{0}$ its connected component and by $\overline{\mathrm{E}}_{0}$ the universal covering of $\mathrm{E}_{0}\left(\overline{\mathrm{E}}_{0}=\right.$ semi-direct product $\mathrm{R}_{\square}^{3} \mathrm{SU}(2)$ of $\mathbf{R}^{3}$ by $\left.\mathrm{SU}(2)\right)$; finally we denote by $\theta$ the surjective group homomorphism :

$$
\overline{\mathrm{E}}_{0} \stackrel{\theta}{\rightarrow} \mathrm{E}_{0} \text {. }
$$

2. Nematics. - As a first illustration, we consider a case already dealt with in ref. [1] in order to show that we do reach the same conclusions. Nematic phases are invariant by translations but not by rotations because the non-spherical molecules of these liquids are oriented (at least partially) so the thermodynamic properties of the nematic phase are described by a quadrupole (symmetric tensor with null trace of $\mathrm{O}(3))$. There are two types of orbits :

$\mathrm{E}(3) /\left(\mathrm{R}_{\square}^{3}\left(\mathrm{D}_{\infty \mathrm{h}}\right)\right) \sim \mathrm{O}(3) / \mathrm{D}_{\infty \mathrm{h}} \sim \mathrm{SO}(3) / \mathrm{D}_{\infty} \sim \mathrm{P}(2, \mathrm{R})$

(the projective real plane) and

$$
\mathrm{E}(3) /\left(\mathrm{R}_{\square}^{3} \mathrm{D}_{2 \mathrm{~h}}\right) \sim \mathrm{O}(3) / \mathrm{D}_{2 \mathrm{~h}} \sim \mathrm{SO}(3) / \mathrm{D}_{2} .
$$

The first kind of orbit corresponds to uniaxial nematics with the homotopy $\pi_{0}=0, \pi_{1}=Z_{2}, \pi_{2}=\mathrm{Z}$ as found in ref. [1]. The second kind of orbits would correspond to biaxial nematics (the quadrupole has three unequal axes) and by a computation similar to that in 3 below one finds $\pi_{0}=0, \pi_{2}=0$ (no t.s. point defects in that case !) and $\pi_{1}=\mathrm{Q}$ the quaternion group (i.e., the group generated by the $i \sigma_{k}$ where $\sigma_{\mathrm{k}}$ are the Pauli matrices); $\pi_{1}$ is not abelian and has five conjugation classes : 1 (the perfect state), $-1, \pm i \sigma_{1}, \pm i \sigma_{2}, \pm i \sigma_{3}$.

3. Crystal. - We can now sketch the classification of the t.s. defects in a crystal. Let $\mathrm{H}$ be the crystallographic group of the crystal under consideration (it belongs to one of the 230 crystallographic classes) : $\mathrm{H}$ is a discrete subgroup of $\mathrm{E}(3)$ such that the orbit $\mathrm{E}(3) / \mathrm{H}$ is compact. Since $\mathrm{E}(3)$ is a covering space of the orbit :

$$
r>1, \quad \pi_{r}(\mathrm{E}(3) / \mathrm{H})=\pi_{r} \mathrm{E}(3)
$$

(see e.g., ref. [2]), so for any crystal

$$
\pi_{2}=0, \quad \pi_{3}=\mathrm{Z}
$$

(see refs. [3] and [4] respectively).
Let $\mathrm{H}_{0}=\mathrm{H} \cap \mathrm{E}_{0}$. If $\mathrm{H}=\mathrm{H}_{0}$, the point group $\mathrm{H} / \mathrm{Z}^{3}$ does not contain reflections, so the orbit $\mathrm{E}(3) / \mathrm{H}$ has two connected components i.e., $\pi_{0}=Z_{2}$; the only other case is when $\mathrm{H}_{0}$ is a subgroup of index two of $\mathrm{H}$; then the orbit is connected and $\pi_{0}=0$. To summarize

$$
\pi_{0}=\mathrm{Z}_{2} \text { if } \mathrm{H} \subset \mathrm{E}_{0}, \quad \pi_{0}=0 \text { otherwise } .
$$

When $\pi_{0}=z_{2}$, there can be wall defects; they are a special type of twin boundary, the twin by reticular merihedry in the terminology of G. Friedel [5]. If $\pi_{0}=Z_{2}$, the two connected components of the orbit are homeomorphic so, in all cases, to compute $\pi_{1}(\mathrm{E} / \mathrm{H})$ we need only to consider $\mathrm{E}_{0} / \mathrm{H}_{0}$ which is homeomorphic to the orbit $\overline{\mathrm{E}}_{0} / \theta^{-1}\left(\mathrm{H}_{0}\right)$; since $\overline{\mathrm{E}}_{0}$ is simply connected

$$
\pi_{1}=\theta^{-1}\left(\mathrm{H}_{0}\right) \text { where } \mathrm{H}_{0}=\mathrm{H} \cap \mathrm{E}_{0} .
$$

In general this group is not abelian, so isolated line singularities in a crystal can be classified by the conjugation classes of $\theta^{-1}\left(\mathrm{H}_{0}\right)$, the covering in $\overline{\mathrm{E}}_{0}$ of the crystallographic group without reflections.

4. What happens when two t.s. defects of the same dimension coalesce ? If each one is described by an element of an abelian group, they combine according to the (abelian) group law. For instance any two wall singularities in a crystal (twin boundaries) annihilate, as do two t.s. line defects in uniaxial nematics and as two vortices in phase $\mathrm{A}$ of ${ }^{3} \mathrm{He}$ should do, as was predicted in ref. [1]. If two isolated line defects are described by conjugation classes of $\pi_{1}$, the composition law of these conjugation classes is that of an abelian algebra (which is the centre of the group algebra). However the function $\varphi$, defined in 1 above, yields more information : indeed the $\pi_{1}$ orbit (by $\pi_{1}$ inner automorphisms) of the product $\alpha \beta$ of a pair of elements of $\pi_{1}$ lies in a unique conjugation class, which is independent of the order since $\alpha \beta$ and $\beta \alpha$ are conjugated : $\alpha \beta=\alpha(\beta \alpha) \alpha^{-1}$.

5. The two following remarks will help the interpretation of paragraph 1 .

a) The local states of non-perfect media are not simply obtained from each other by Euclidean displacements, but more generally by linear or even by differentiable transformations. In the case of crystals, consider for instance the figures of ref. [6] Ch. 3 . So the function $\varphi$ defined in 1 above takes its values in $\left(\right.$ Diff $\left.R^{d}\right) / H$ where Diff $R^{d}$ is the group of diffeomorphisms of the $d$-dimensional Euclidean space. This does not change our conclusions : indeed, using the work of Stewart [7], we obtain

$$
\pi_{r}\left(\operatorname{Diff} R^{n} / \mathrm{H}\right)=\pi_{r}(\mathrm{E}(n) / \mathrm{H}) .
$$

(It is for pedagogical reasons that we first introduced the Euclidean group.) 
b) One can also consider instead of $\mathrm{G} / \mathrm{H}$ a family of orbits $(\mathrm{G} / \mathrm{H})(\eta)$ corresponding to the different values of the Landau [7] (scalar) order parameter $\eta$. This positive parameter decreases as a function of $T$ and reaches zero at the temperature $T_{\mathrm{c}}$ of the phase transition to the symmetrical phase. For $\eta>0$ all orbits are homeomorphic and the family

$$
\left\{(\mathrm{G} / \mathrm{H})(\eta), \quad 0<\eta_{1} \leqslant \eta \leqslant \eta_{2}\right\}
$$

is the topological product of $\mathrm{G} / \mathrm{H}$ and a line segment, so it has the homotopy of $\mathrm{G} / \mathrm{H}$. However, when the fluctuations of $\eta$ to the value $\eta=0$ can no longer be neglected (when $T$ goes nearer to $T_{\mathrm{c}}$ i.e. usually when $T$ increases) the manifold of states to be considered is the union $\left\{(\mathrm{G} / \mathrm{H})(\eta), 0 \leqslant \eta \leqslant \eta_{2}\right\}$. It is contractible into the point $\eta=0$, so its homotopy is trivial i.e., the defects become unstable (e.g. annealing process).

Acknowledgments. - One of us (L. M.) is grateful to the Theoretical Physics Division of Los Alamos Laboratories where he began this work, and to Professors Arthur G. Wasserman and Christopher Zeeman for very stimulating discussions; A. W. gave us the idea of remark V.a.

After this work was finished, our attention was called to the paper of D. Rogula in Trends in applications of pure Mathematics to Mechanics, p. 311-331, edited by G. Fichera, Pitman Publ. London 1976. This paper proposes homotopy for classification of defects and gives some applications to Bravais lattices.

\section{References}

[1] Kléman, M. and Toulouse, G., J. Physique Lett. 37 (1976) L-149.

[2] Steenrod, N., The Topology of Fiber Bundles (Princeton Univ. Press) 1957.

[3] CARTAN, E., La topologie des espaces représentatifs des groupes de Lie, Act. Sci. Ind. 358 (Hermann Paris) 1936.

[4] BotT, R., Proc. Nat. Acad. Sci. U.S. 40 (1954) 586.
[5] FRIEDEL, G., Leçons de cristallographie (Berger-Levrault, Paris) 1926.

[6] Nabarro, F. R. N., Theory of crystal dislocations (Clarendon Press Oxford) 1967

[7] Stewart, T. E., Proc. Am. Math. Soc. 11 (1960) 559.

[8] Landau, L. and Lifschitz, E., Statistical Physics (Pergamon) 1958. 